\title{
A high performance ultra-wideband low cost SMA-to-GCPW transition
}

\author{
Ding Xu ${ }^{1 \text { a) }}$, Zhengpeng Wang ${ }^{1}$, Yi Wang ${ }^{2}$, and Jianhua Wu ${ }^{1}$ \\ ${ }^{1}$ School of Electronic and Information Engineering, Beihang University, \\ Beijing, 100191, China \\ ${ }^{2}$ Department of Engineering Science, University of Greenwich, Kent, ME4 4TB, UK \\ a)xuding08@gmail.com
}

Abstract: This letter presents a novel low cost through-the-wall SMA connector and the transition structures from the SMA to a grounded coplanar waveguide (GCPW). The SMA connector has two short metal legs extended from the outer conductor used to reduce the discontinuity of the transition. The parameters of the GCPW are designed to match the geometry of the coaxial. A matching stub is introduced in the center conductor line to further improve the performance of the transition. A prototype device is developed and measured. The measurement results show the return loss of the proposed transition is better than $20 \mathrm{~dB}$ up to $26.5 \mathrm{GHz}$.

Keywords: transition, SMA, GCPW, K-band

Classification: Microwave and millimeter-wave devices, circuits, and modules

\section{References}

[1] R. N. Simons: Coplanar Waveguide Circuits, Components, and Systems (Wiley, New York, 2004) 1.

[2] E. England: IEEE Trans. Microw. Theory Techn. 24 (1976) 47 (DOI: 10.1109/ TMTT.1976.1128765).

[3] M. L. Majewski, et al.: IEEE Trans. Microw. Theory Techn. 29 (1981) 799 (DOI: 10.1109/TMTT.1981.1130450).

[4] R. Eisenhart: IEEE MTT-S Dig. 78 (1978) 318 (DOI: 10.1109/MWSYM.1978. 1123876).

[5] R. Neidert: Microw. J. 26 (1983) 93.

[6] J.-C. Cheng, et al.: IEEE Trans. Microw. Theory Techn. 59 (2011) 1468 (DOI: 10.1109/TMTT.2011.2123911).

[7] T. Kamei, et al.: IEICE Trans. Electron. E90-C (2007) 2030 (DOI: 10.1093/ ietele/e90-c.10.2030).

[8] B. Rosas: Southwest Microwave, Inc., Tempe, AZ (2007) http://www. southwestmicrowave.com.

[9] O. Moravek, et al.: IEEE Trans. Microw. Theory Techn. 61 (2013) 2956 (DOI: 10.1109/TMTT.2013.2272380).

[10] O. Moravek and K. Hoffmann: Electron. Lett. 48 (2012) 1003 (DOI: 10.1049/ el.2012.0772). 
[12] X. Wu, et al.: IEEE Trans. Adv. Packag. 31 (2008) 182 (DOI: 10.1109/TADVP. 2007.914962).

\section{Introduction}

SMA connectors are widely used in microwave circuits to connect to planar transmission lines such as microstrip, coplanar waveguide (CPW) and grounded coplanar waveguide (GCPW). Among them, the GCPW has been increasingly used in the current RF/microwave circuit design due to its lower radiation loss and lower dispersion at high frequencies as compared with a microstrip line [1]. The GCPW also has the advantages of easy integration with surface mount devices and the flexibility to control the impedance by adjusting the ratio between the center conductor width and the slot, as in a CPW. Normally SMA connectors operate below $18 \mathrm{GHz}$ whereas $3.5 \mathrm{~mm}$ or $2.4 \mathrm{~mm}$ connectors are employed at frequency bands higher than $18 \mathrm{GHz}$. With the development of MMIC technologies into increasingly high frequencies, high-frequency connectors are demanded for both industrial and consumer applications. As an example, $24 \mathrm{GHz}$ has been widely used in short range vehicle collision avoidance radars and industrial level-measurement radars. These applications are often cost sensitive, so low cost and reliable connectors and their transitions to planar transmission lines are highly desired.

A lot of research results of coaxial-to-microstrip transitions have been presented in the past. [2] and [3] made the theoretical analysis and numerical modeling for the general coaxial-to-microstrip transitions. [4, 5, 6, 7] presented some new SMA structures for the extension of the transition's bandwidth and the improvement of the return loss. [8] compared the transmission characteristics of GCPW and microstrip at high frequencies and introduced a tapered impedance matching section to achieve better return loss. However, most of these methods rely on precise mechanical fabrication and complex assembling, which implies higher cost.

In this letter, a novel low cost through-the-wall SMA connector and the design of its transition to GCPW are presented. The novel SMA connector has two short metal legs extended from the outer conductor of the coaxial that are used to reduce the discontinuity of the transition. A matching stub is added in the GCPW to further improve the return loss and insertion loss. It is shown that the proposed transition has an insertion loss less than $1 \mathrm{~dB}$ and return loss better than $20 \mathrm{~dB}$ over the frequency range from $0.1 \mathrm{GHz}$ to $26.5 \mathrm{GHz}$. Compared with the conventional SMA-to-GCPW transitions, over $60 \%$ increase of the bandwidth is achieved without requiring significant modification on the SMA connector and the installation.

\section{Proposed design}

There are two common sizes of the universal inexpensive SMA connectors. One has the center pin diameter of $1.27 \mathrm{~mm}$ and inner diameter of the outer conductor of $4.11 \mathrm{~mm}$. The other one is $0.8 \mathrm{~mm}$ and $2.68 \mathrm{~mm}$ respectively. The latter connector has a cutoff frequency of its first higher order mode at around $37 \mathrm{GHz}$ whereas the 
former has it at $25.1 \mathrm{GHz}$. In this work, the second type of SMA connector is adopted for the implementation of the SMA-to-GCPW transitions.

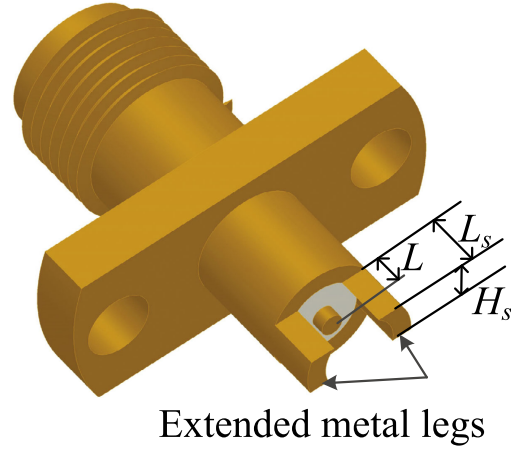

(a)

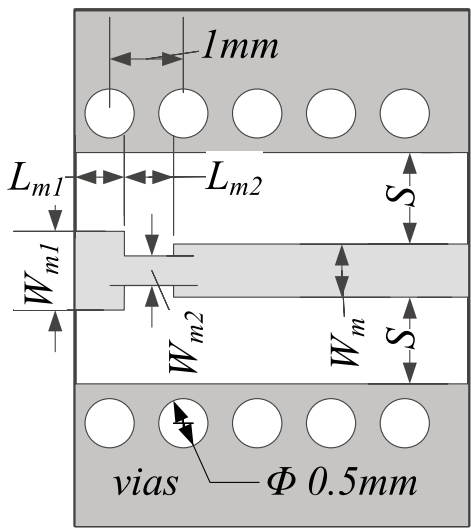

(b)

Fig. 1. (a) The proposed SMA connector with two extended metal legs; (b) the corresponding GCPW with an in-line matching stub.

The GCPW line is fabricated on Rogers RT Duroid 4350b substrate with a relative dielectric constant of 3.66 and a loss tangent of 0.004 . The metal thickness is $17 \mu \mathrm{m}$ and the substrate thickness is $254 \mu \mathrm{m}$. The geometry of the modified SMA connector and the GCPW line is shown in Fig. 1. For the GCPW, the diameter of the vias is $0.5 \mathrm{~mm}$ and the space between them is $1 \mathrm{~mm}$, so the corresponding cutoff frequency is $78 \mathrm{GHz}$. To minimize discontinuity, the distance between the two inplane grounds is set to match the distance between the outer conductors of the coaxial at the joints between the SMA and the GCPW. Based on this requirement, the center line width of the GCPW $\mathrm{W}_{\mathrm{m}}$ and the gap between the center conductor and the ground plane, $\mathrm{S}$, are determined.

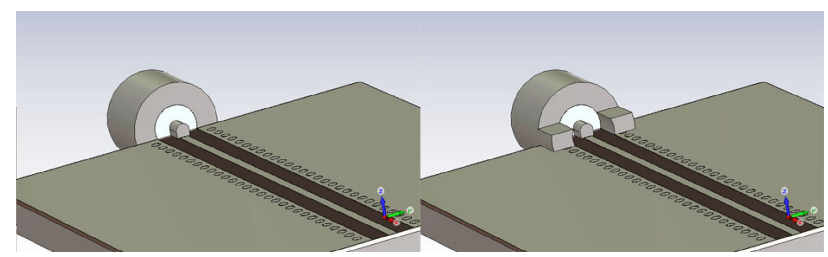

(a)

(b)

Fig. 2. (a) Conventional transition; (b) The transition with the proposed SMA connector but without matching stub in the GCPW.

To achieve a good transition efficiency, the radiation of the connector should be limited by increasing the coupling region between the transitions $[6,9,10]$. Two metal legs are introduced to reduce the abrupt changes of the electromagnetic field distributions from the SMA connector to the GCPW line. The conventional coaxialto-GCPW transition without the metal legs is shown in Fig. 2(a). For the proposed SMA connector, the two metal legs are soldered onto the two ground planes as shown in Fig. 2(b). The center line width, Wm1, is $0.8 \mathrm{~mm}$ which is the same as 
the center pin diameter of the SMA as shown in Fig. 1(b). The length Lm1 of the contact pad in the GCPW is the same as the length $\mathrm{L}$ of the coaxial pin of the SMA.

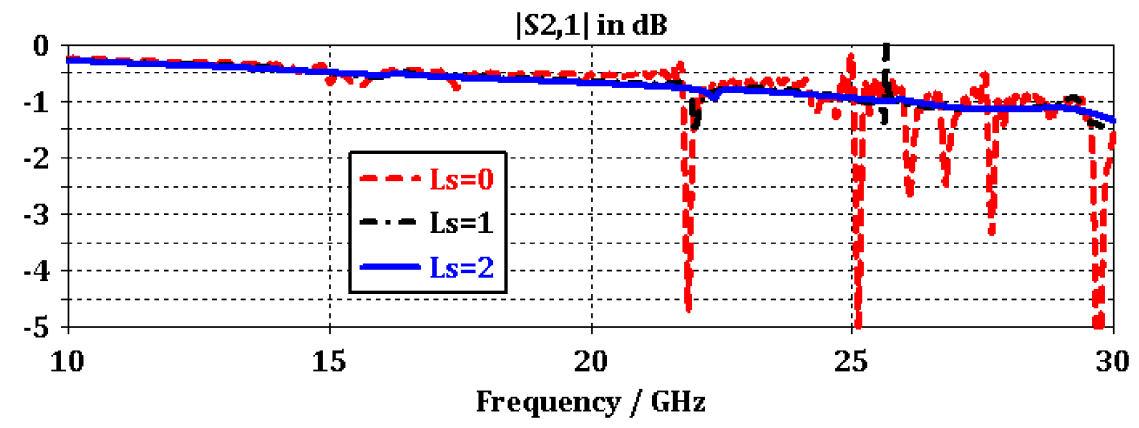

Fig. 3. The transmission responses with different lengths of the extended metal legs

(a)

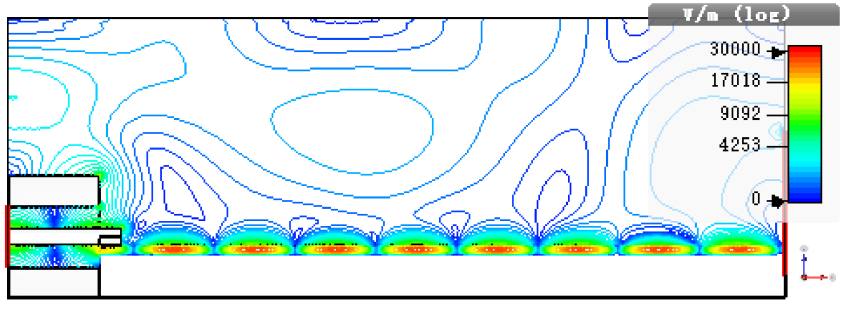

(b)

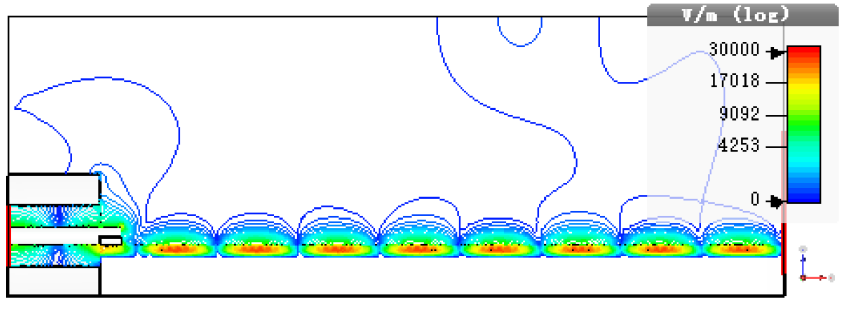

(c)

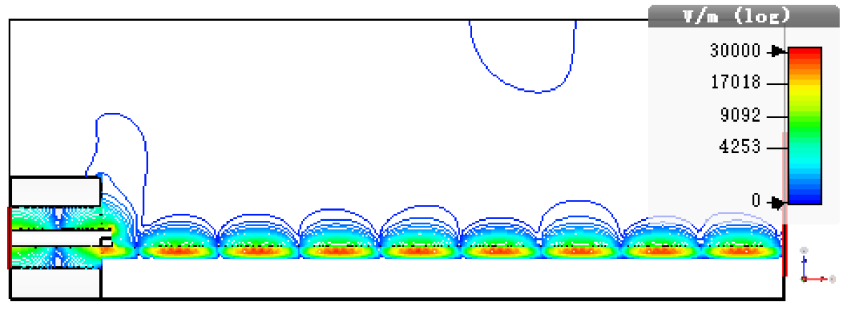

(d)

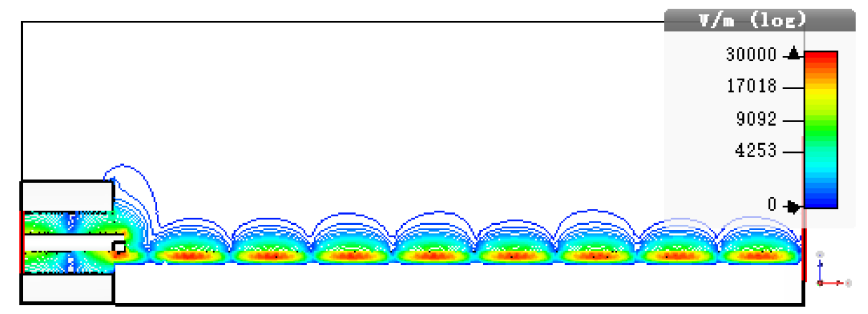

Fig. 4. Simulated E-field radiation from the transition at $25 \mathrm{GHz}$ :

(a) Conventional design; (b) $\mathrm{L}=1 \mathrm{~mm}$ with $\mathrm{Ls}=2 \mathrm{~mm}$; (c) $\mathrm{L}=0.5 \mathrm{~mm}$ with $\mathrm{Ls}=2 \mathrm{~mm}$; (d) Configuration-(c) with an additional GCPW matching stub. 
electrically small at low frequency, the size optimization of the metal legs focuses on the performance at high frequency bands. If $\mathrm{H}_{\mathrm{s}}$ and $\mathrm{L}$ is set to $1 \mathrm{~mm}$, for example, the length of the metal legs $L_{s}$ should be longer than $0.2 \lambda$ at the highest operating frequency. The simulation results of the transmission response with different $\mathrm{L}_{\mathrm{s}}$ are displayed in Fig. 3. The simulation is conducted by CST ${ }^{\mathrm{TM}}$ Microwave Studio software. As can be seen from the graph, the extended metal legs reduced the ripples in the transmission response as compared with the conventional SMA connectors. Most of the resonance points from the conventional design are eliminated. The radiation from the transitions with and without the extended metal legs are also simulated and the results are displayed in Fig. 4. The distribution of the electric field intensity of the conventional transition is displayed in Fig. 4(a) and the radiation from the proposed transition is displayed in Fig. 4(b), where $\mathrm{L}$ is $1 \mathrm{~mm}$ and the $\mathrm{L}_{\mathrm{s}}$ is $2 \mathrm{~mm}$. It is observed that the proposed SMA suppresses the radiated energy and most of the electric fields are constrained near the PCB.

At the upper frequency range, the exposed center pin of the SMA causes significant radiation [9], so reducing the length of the center pin limits the unwanted radiation from the connector. The simulation results show that the shorter the center pin, the smaller the VSWR is. In consideration of the ease of soldering and the reliability of the connection, the center pin is chosen to be $0.5 \mathrm{~mm}$. As shown in Fig. 4(c), when $\mathrm{L}$ is $0.5 \mathrm{~mm}$ the radiation is further reduced.

The center pin of the SMA and the GCPW line welded together presents capacitive impedance characteristics at high frequencies. Additional inductive reactance is required to offset the capacitive reactance and achieve good impedance matching [11]. In this work, a high impedance inductive matching line is used in series with the center pin of the SMA to cancel out the capacitive reactance. As can be seen from Fig. 1(b), the dimensions of $\mathrm{L}_{\mathrm{m} 2}$ and $\mathrm{W}_{\mathrm{m} 2}$ determine the inductive matching effect. The optimized dimensions after parameter studies and layout optimization are listed in Table I.

Table I. Parameter list

\begin{tabular}{lccccccccc}
\hline parameters & $\mathrm{H}_{\mathrm{s}}$ & $\mathrm{L}_{\mathrm{s}}$ & $\mathrm{L}$ & $\mathrm{S}$ & $\mathrm{W}_{\mathrm{m}}$ & $\mathrm{W}_{\mathrm{m} 1}$ & $\mathrm{~L}_{\mathrm{m} 1}$ & $\mathrm{~W}_{\mathrm{m} 2}$ & $\mathrm{~L}_{\mathrm{m} 2}$ \\
\hline Value $(\mathbf{m m})$ & 1 & 2 & 0.5 & 1 & 0.59 & 0.8 & 0.5 & 0.3 & 0.5 \\
\hline
\end{tabular}

The simulated $\mathrm{S}_{11}$ curves of the transition with and without the matching stub are shown in Smith chart in Fig. 5(a). From this chart, it can be observed that the proposed matching stub achieves very good matching over the whole frequency band from 0 to $30 \mathrm{GHz}$. The unwanted field radiation from the proposed transition is eliminated at $25 \mathrm{GHz}$ as can be seen from Fig. 4(d). Fig. 5(b) shows the simulated frequency responses of the proposed SMA-to-GCPW transition. The $\mathrm{S}_{11}$ is less than $-20 \mathrm{~dB}$ and $\mathrm{S}_{21}$ is better than $-0.5 \mathrm{~dB}$ up to $26.5 \mathrm{GHz}$. Compared with the conventional transition, the proposed transition gives significantly improved transmission efficiency over an increased working bandwidth. 


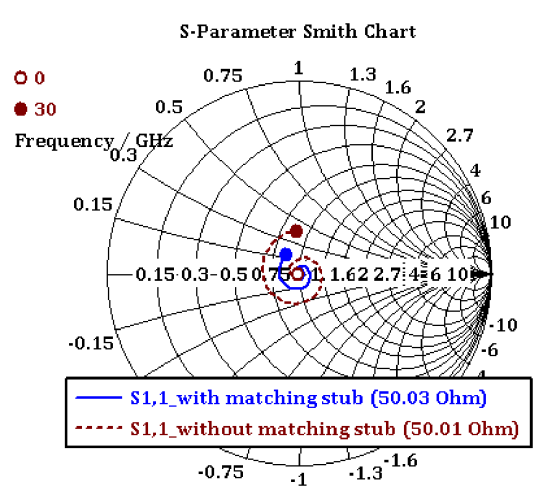

(a)

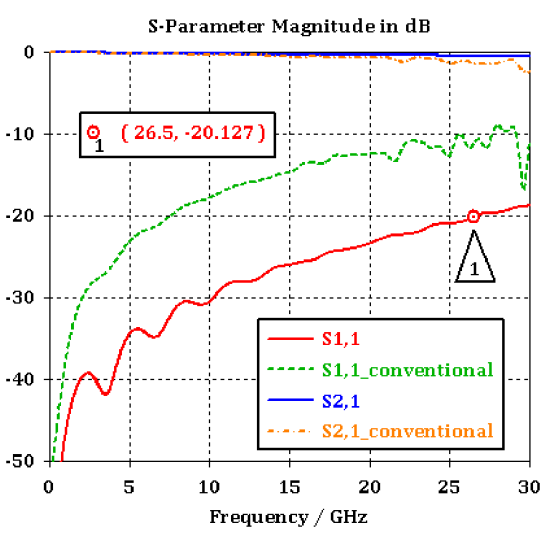

(b)

Fig. 5. The simulated frequency responses of the optimized design: (a) Smith chart, (b) S-parameters.

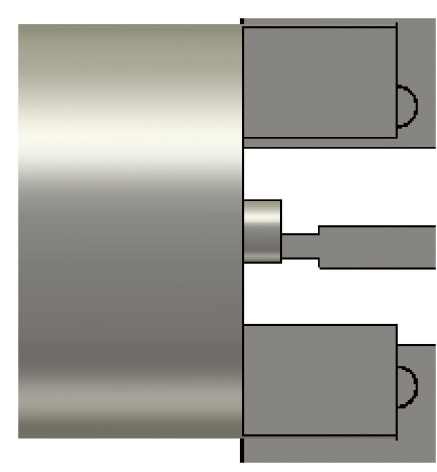

(a)

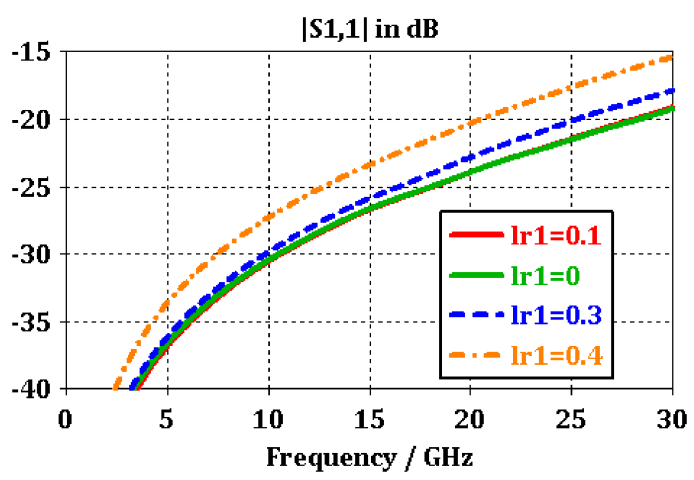

(b)

Fig. 6. (a) Misalignment model. (b) Simulated $S_{11}$ results of the proposed transition subjected to misalignment errors.

The influence of misalignment in the assembly process on the performance of the proposed transition is studied. As shown in Fig. 6(a), there is misalignment error between the center pin of the proposed SMA and the GCPW line. The distance between the centers of the two line is $\operatorname{lr} 1$ and the return loss characteristics due to the variation of $1 \mathrm{r} 1$ from 0 to $0.4 \mathrm{~mm}$ are simulated and described in Fig. 6(b). The result shows the misalignment error has little impact on the transmission characteristic until the dimension of misalignment reach 50 percent of the diameter of the center pin of the SMA.

The transition for the GCPW line with smaller gaps is also investigated. Two different dimensions of GCPW line with same impedance of $50 \Omega$ are used to test the performance of the transition. One GCPW line is $\mathrm{S}=0.5 \mathrm{~mm}$ and $\mathrm{W}_{\mathrm{m}}=0.56 \mathrm{~mm}$ and the other one is $\mathrm{S}=0.35 \mathrm{~mm}$ and $\mathrm{W}_{\mathrm{m}}=0.54 \mathrm{~mm}$. The simulated results are shown in Fig. 7(b). We can see that the small gaps have some effects on the S parameters, but the transitions' performances are still fairly good compared with the conventional transition. If needed, a GCPW-to-GCPW transition with different size of gaps can be added to get the best transmission performance. 


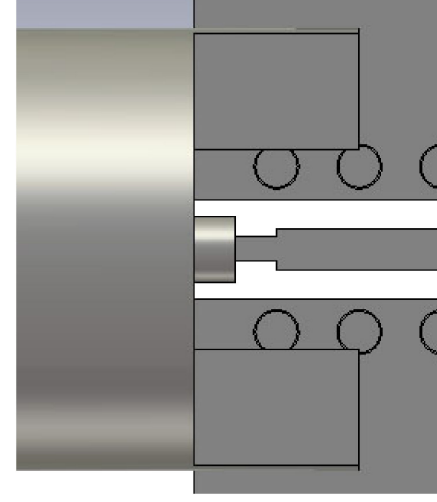

(a)

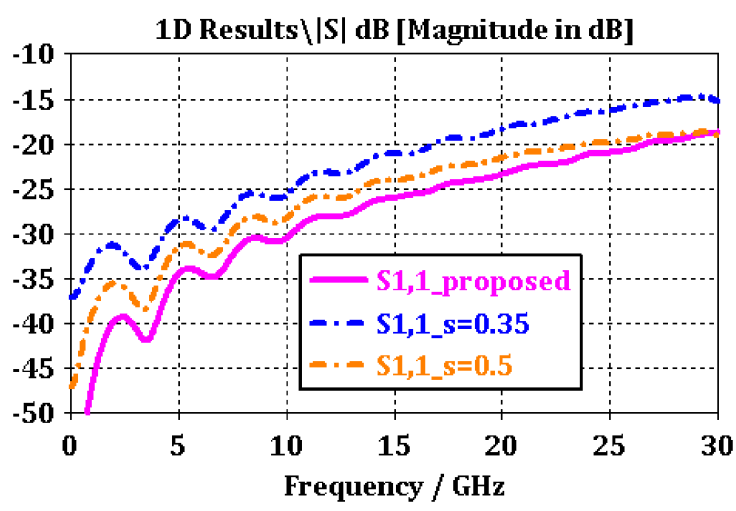

(b)

Fig. 7. (a) GCPW line with small gaps. (b) Simulated frequency responses of the different GCPW-to-SMA transitions

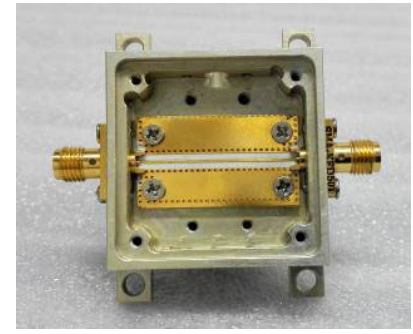

(a)

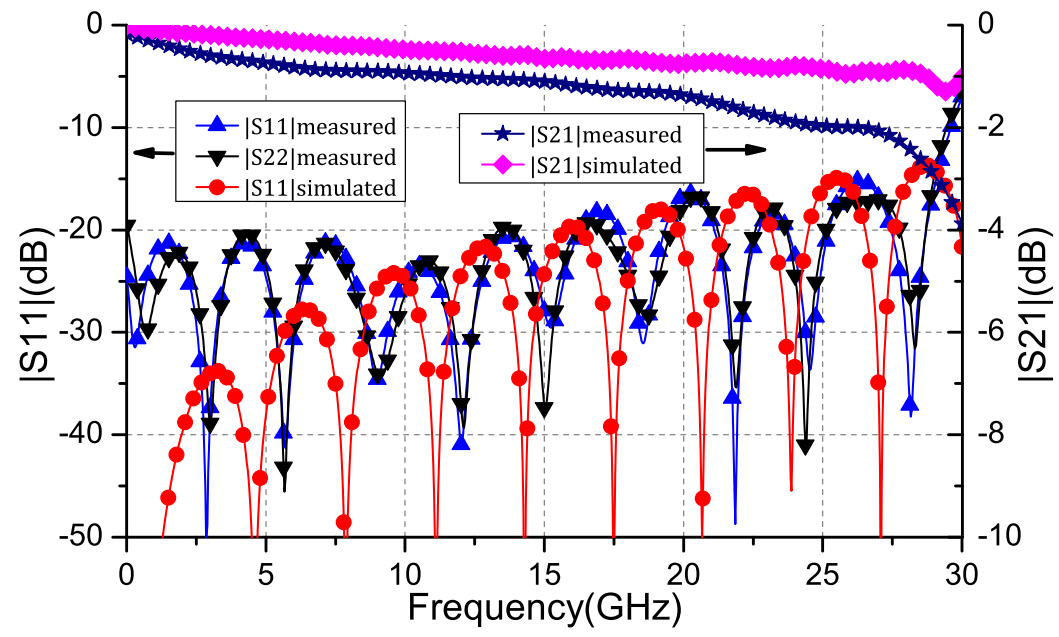

(b)

Fig. 8. (a) Prototype of the back-to-back SMA-to-GCPW transitions. (b) Measured and simulated frequency responses of the back-toback SMA-to-GCPW transitions.

\section{Results and discussion}

A prototype SMA connector with the modified structure has been manufactured. The two short metal legs of the SMA connector are cut from the extended coaxial outer conductor using a milling machine. The SMA-to-GCPW transitions with a back-to-back structure is fabricated to verify the effectiveness of the design. The test structure consists of two proposed SMA connectors and one GCPW printed circuit board with the proposed matching stubs. The GCPW board is $28 \mathrm{~mm}$ long. 
Fig. 8(a) shows the prototype device. It is tested using Agilent PNA E8363B network analyzer. Fig. 8(b) shows the measurement results in comparison with simulations. It can be obtained that the measured $\mathrm{S}_{11}, \mathrm{~S}_{22}$ and $\mathrm{S}_{21}$ curves agree well with the simulated ones. The return loss was better than $15 \mathrm{~dB}$ up to $26 \mathrm{GHz}$ and the transmission loss is less than $2 \mathrm{~dB}$. The measured transmission loss is larger than the simulated one. This is mainly because of the electroless nickel immersion gold (ENIG) technology is used for the PCB surface plating. Its conductivity is lower than pure copper [12]. So compared with the simulation using pure copper, the actual conductor loss is higher. In general, the measured results show that the proposed transition has excellent transmission characteristics in ultra wideband frequency.

\section{Conclusion}

A novel and low cost SMA connector with extended metal ground is presented. It has been demonstrated that this modified SMA structure improves the transmission performance at high frequencies. An in-line matching GCPW stub is also proposed for the SMA-to-GCPW transition circuit design, which helps to further minimize the reflection and improve the performance at the upper frequency range. The measured results agree well with the simulated ones. In addition, the proposed transition has similar installation of welding as the conventional transition. With its broadband performance, simple structure and low cost, such a SMA-to-GCPW transition is most suitable for industrial radar and wireless communication applications up to $\mathrm{K}$ band. 